Check for updates

Cite this: J. Mater. Chem. C, 2019, 7, 2987

Received 16th November 2018 Accepted 7th February 2019

DOI: $10.1039 / c 8 t c 05774 \mathrm{e}$

rsc.li/materials-c

\title{
Organic electrochemical transistors from supramolecular complexes of conjugated polyelectrolyte PEDOTS $\uparrow$
}

\author{
Chiara Musumeci, (D) *a Mikhail Vagin, ${ }^{b}$ Erica Zeglio, ${ }^{a}$ Liangqi Ouyang, (DD ${ }^{a}$ \\ Roger Gabrielsson ${ }^{\mathrm{b}}$ and Olle Inganäs*a
}

\begin{abstract}
Counterion exchange strategies are used to modify the hydrophilic character of the self-doped conjugated polyelectrolyte PEDOTS. The supramolecular complexes, soluble in organic solvents, are suitable to fabricate finely performing thin active layers in organic electrochemical transistors (OECTs). We demonstrate that ionic transport in these PEDOTS based complexes, thus their performance in OECT devices, is governed by a delicate balance among degree of doping, wettability and porosity, which can be controlled by a precise tuning of the polyelectrolyte/hydrophobic counterion ratio. We also show that the device operation can be modulated by varying the composition of the aqueous electrolyte in a range compatible with biological processes, making these materials suitable candidates to be interfaced with living cells.
\end{abstract}

\section{Introduction}

Conjugated polyelectrolytes are a class of (semi)-conducting polymers possessing charged groups covalently attached to the conjugated backbone. By enabling both electronic and ionic transport, they bridge the gap between electronics and biology, allowing the development of intimate bioelectronics interfaces. ${ }^{1}$ The charged side groups, making these materials soluble in polar solvents, allow for the incorporation of water molecules and mobile ions into the polymer matrix, leading to improved ionic conductivity with respect to conjugated polymers having hydrophobic side groups. Some conjugated polyelectrolytes are self-doped, meaning that the charged side groups act as counterions to balance the positive charges formed in the conjugated backbone upon oxidation. ${ }^{2}$ PEDOTS is a conjugated polyelectrolyte in which alkoxysulfonate side chains are covalently bound to a poly(3,4-ethylenedioxythiophene) (PEDOT) backbone. The negatively charged sulfonate groups render PEDOTS highly water soluble and stabilize the positive charges on the polymer backbone in its pristine oxidized conductive state, thus making PEDOTS a self-doped polymer with conductivity of tens of $\mathrm{S} \mathrm{cm}^{-1} \cdot{ }^{3}$ Changes in the level of oxidation also change PEDOTS

\footnotetext{
${ }^{a}$ Biomolecular and Organic Electronics, Department of Physics Chemistry and Biology, Linköping University, SE-58183 Linköping, Sweden.

E-mail: chiara.musumeci@liu.se, olle.inganas@liu.se

${ }^{b}$ Laboratory of Organic Electronics, Department of Science and Technology, Linköping University, SE-60174 Norrköping, Sweden

$\dagger$ Electronic supplementary information (ESI) available. See DOI: 10.1039/ c8tc05774e
}

conductivity, thus making it a suitable active material for the fabrication of functional devices, such as organic electrochemical transistors (OECTs). ${ }^{4-6}$ In a typical OECT, a gate voltage drives ions from an electrolyte into the bulk of the conjugated polymer, thus modulating its redox state and conductivity. Low voltage operation, high transconductance, and compatibility with operation in aqueous environment (e.g. biological media) make OECTs ideal devices to be coupled and interfaced with living systems and to work as active amplifying ion-to-electron transducers, ultimately increasing the signal-to-noise ratio with respect to traditional electrodes used for electrophysiology. ${ }^{7,8}$ The manipulation of the hydrophobic/hydrophilic character of these electrode materials may also have potential for the solid/ liquid/gas interfaces found in fuel cells driven with gases.

The charged side chains of PEDOTS can also be exploited to fabricate new materials by supramolecular interactions. The negative sulfonate groups can in fact interact electrostatically with positively charged moieties, creating supramolecular complexes with new functionalities. This strategy has been specifically used for the fabrication of conductive fibers from biotemplates such as protein fibers ${ }^{9-12}$ and DNA, ${ }^{13}$ for the fabrication of multilayered electrochromic films ${ }^{14,15}$ and conductive hybrid nanocomposites. ${ }^{16}$ By using counterion exchange strategies, i.e. by replacing small counterions with ions bearing long hydrophobic chains, it is also possible to modify the hydrophilic character of PEDOTS, creating new materials which are soluble in organic solvents and can form films that are stable in aqueous electrolytes without the need of crosslinking. ${ }^{17} \mathrm{We}$ have also shown that these materials can be used as active layers 
in OECTs, ${ }^{17}$ can be inserted into lipid membranes and used to modify biological functions in living cells. ${ }^{18}$ The OECTs based on these hydrophobic complexes, however, required relatively concentrated aqueous electrolytes (e.g. $1 \mathrm{M} \mathrm{KCl})$ to be operational. We anticipate indeed that they show poor or null switching ability at electrolyte concentrations around $0.1 \mathrm{M}$ (or lower), corresponding to physiological conditions where cells and biological systems function. Here we investigate the parameters affecting the operation of PEDOTS based hydrophobic complexes in OECTs, aiming to get guidelines for the design of these materials and their use in biologically compatible environment.

\section{Results and discussion}

PEDOTS : DOA complexes having different compositions were obtained by mixing PEDOTS (from $1 \mathrm{mg} \mathrm{mL}^{-1}$ solutions) with dioctylammonium (DOA) at different molar ratios, namely $1: 10,1: 1$ and $2: 1$, with respect to the EDOTS monomer. Scheme and images relative to the different preparation steps are shown in Fig. 1. In the three cases the formation of the complex resulted in a blue precipitate, insoluble in water (Fig. 1d), which could be readily dissolved in chloroform/ methanol 1:1 v/v (Fig. 1e). At first glance, the three solutions appeared slightly different in color. While the PEDOTS:DOA 1:10 solution showed a light purple color, the other two appeared light blue. This color change, from purple to pale blue can be observed when PEDOTS is switched from the neutral to the oxidized state, as a consequence of the decrease of absorption of the $\pi-\pi^{*}$ transition at around $600 \mathrm{~nm} .^{3,12}$ Accordingly, UV-Vis absorption spectra of the three complexes (Fig. 1f) showed a higher level of dedoping for the 1:10 complex with respect to the other two complexes, demonstrated by the increased absorption at around $560 \mathrm{~nm}$. The increased dedoping is likely due to a larger amount of DOA cations interacting with the sulfonate side groups of PEDOTS during the complex formation, and making them unavailable to stabilize the positive charges on the backbone. On the other side, the PEDOTS:DOA 1:1 showed the lowest absorption at $560 \mathrm{~nm}$, indicating a higher level of oxidation of the PEDOTS backbone. PEDOTS: DOA 2:1 showed a similar spectrum to the 1:1 sample, indicating similar doping level.

Electrochemical transistors having PEDOTS:DOA 1:10 films as an active layer showed good switching properties when using electrolyte at high ionic strength, i.e. $1 \mathrm{M} \mathrm{KCl}$, but scarce or null operation in $0.1 \mathrm{M} \mathrm{KCl}$, as shown in Fig. 2a. Increasing the PEDOTS to DOA ratio to $1: 1$ led to improved operation in $0.1 \mathrm{M} \mathrm{KCl}$, suggesting that a higher content of hydrophobic counterions in PEDOTS : DOA 1:10 may likely hinder both the ionic and electronic transport. However, the use of the PEDOTS : DOA 2:1 did not seem to enable better device performance. On the contrary, these devices remained in the non-conductive state in the voltage range investigated (Fig. $2 \mathrm{~b}$ ).

The ionic permeability of the different films at different ionic strengths was evaluated through cyclic voltammetry by using potassium ferricyanide as a redox probe (Fig. 3). The blocking properties of the PEDOTS:DOA 1:10 at low ionic strength $(0.1 \mathrm{M} \mathrm{KCl})$ are demonstrated by the decreased current with respect to the one observed on bare gold microelectrodes. The increased currents in the PEDOTS : DOA $1: 1$ and $2: 1$ seem to indicate instead an improved ionic transport in these films. At higher ionic strengths, i.e. at electrolyte concentrations of $0.5 \mathrm{M}$ and $1 \mathrm{M}$ (Fig. $3 \mathrm{~b}$ and c), current increases for all film compositions. However, the cyclic voltammograms of Fig. 3 also show increased capacitive background contribution at higher PEDOTS:DOA ratios, as well as electrolyte concentrations, which makes an accurate evaluation of the redox current problematic. ${ }^{19}$ Peak currents, $i_{\mathrm{p}}$, increased linearly with the square root of the scan rate $\left(\nu^{1 / 2}\right)$ as an indication of a diffusionlimited process (Fig. S4, ESI $\dagger$ ). By fitting the $i_{\mathrm{p}} \nu s . \nu^{1 / 2}$ curves to
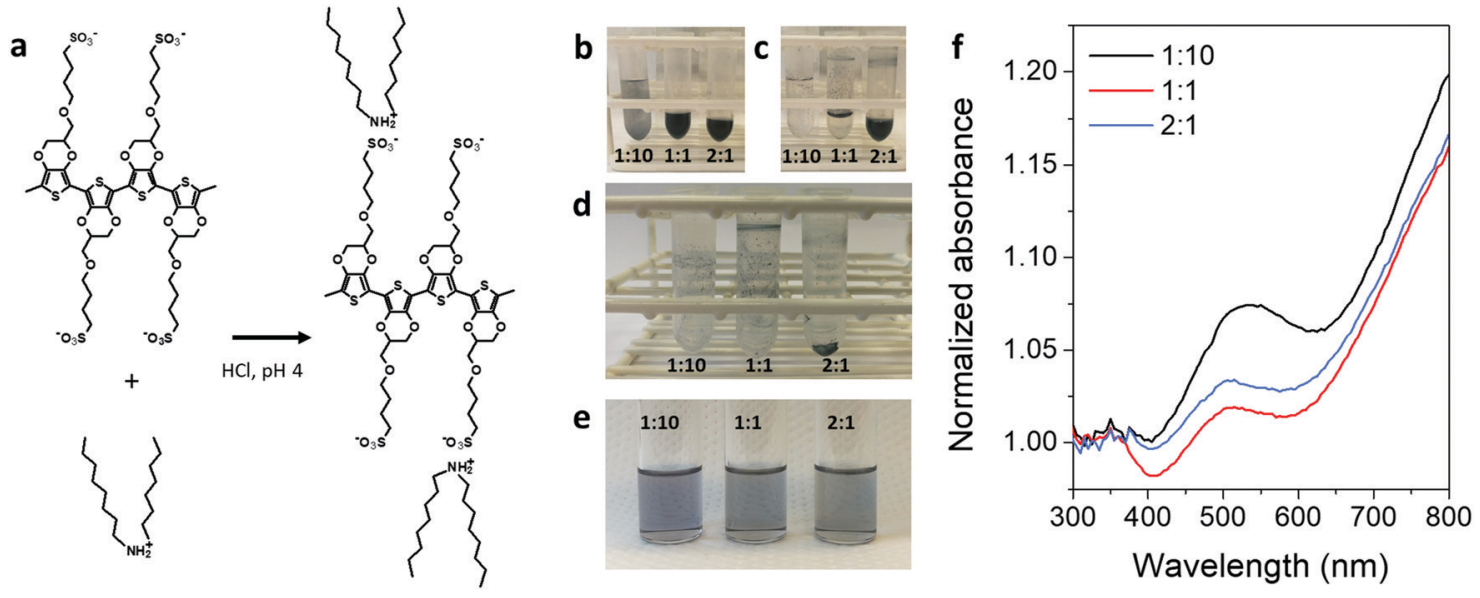

Fig. 1 Preparation of the PEDOTS:DOA complexes. (a) Scheme of the assembly of the complexes from the PEDOTS polyelectrolyte and dioctylammonium salt. (b) Solutions of PEDOTS after addition of the DOA at different PEDOTS/DOA ratios, namely 1:10, 1:1 and 2:1 with respect to the monomer. (c) Precipitation of PEDOTS:DOA hydrophobic complexes from aqueous solutions obtained after vortexing the solutions in (b). (d) Dry PEDOTS:DOA hydrophobic complexes. (e) PEDOTS:DOA solutions in chloroform/methanol. (f) UV-Vis absorbance spectra of PEDOTS:DOA complexes in chloroform/methanol. 

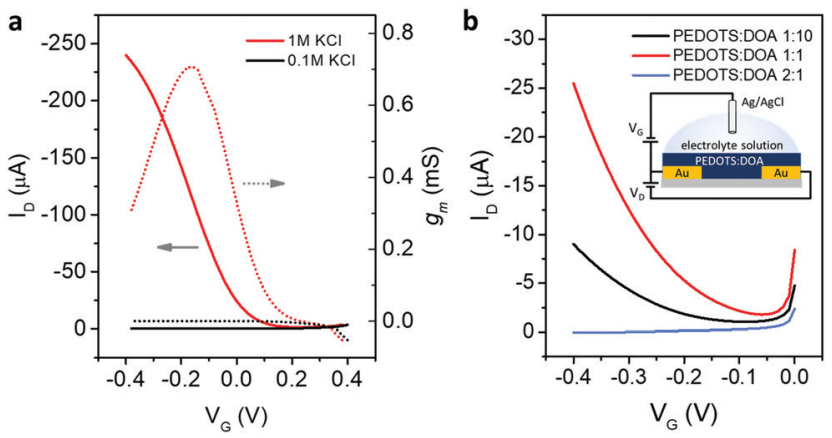

Fig. 2 Electrochemical transistors, OECTs. (a) Transfer characteristics (solid line, left axis) and transconductance (dashed line, right axis) of PEDOTS : DOA $1: 10$ in $0.1 \mathrm{M}$ and $1 \mathrm{M} \mathrm{KCl}$. (b) Transfer characteristics of PEDOTS : DOA $1: 10,1: 1$ and $2: 1$ in $0.1 \mathrm{M} \mathrm{KCl}$. The scheme of the devices is shown in the inset in (b). Additional curves for these devices are shown in Fig. S1 and S2, ESI. $\dagger$

the Randles-Sevcik equation we estimated the effective area in the different electrolyte concentrations (Fig. S1, ESI $\dagger$ ) and observed that while the PEDOTS : DOA 1:10 film showed only a $19 \%$ area increase with respect to the bare $\mathrm{Au}$ electrode in $0.1 \mathrm{M} \mathrm{KCl}$, an increase of $72 \%$ was calculated for the $1: 1$ sample. Moreover, higher electrolyte concentration resulted in a larger increase for both samples, up to $81 \%$ with respect to the dilute solution for the PEDOTS:DOA 1:10 and 113\% for the PEDOTS:DOA 1:1 in $1 \mathrm{M} \mathrm{KCl}$. These values suggest a different degree of swelling and ion uptake of the two samples.

To get more insight on the changes in electrolyte-accessible surface areas with varying the composition, we used a combined characterization through atomic force microscopy (AFM) and electrochemical impedance spectroscopy (EIS). AFM analysis showed a different morphology for the three films of comparable thickness (Fig. 4). Although AFM only images the top surface of the films, some information can be drawn when comparing the topography of the different samples. PEDOTS : DOA $1: 10$ shows a high surface roughness approximately two times larger than the other two films, and a decreasing surface area from the 1:10 to the $1: 1$ and the $2: 1$, as shown in Table 1 . The low value of surface area difference, representing the ratio between the surface area and its projected area, in PEDOTS:DOA $2: 1$, indicate a higher level of compactness of the film, which may be the cause of the hindered ionic transport into the bulk of the film during OECTs operation. The highest roughness of PEDOTS:DOA 1:10 should instead favor ionic transport and enhance operation in these films, since the capacitance of an electrode is generally directly dependent on its surface area. However, capacitance can also depend on other parameters such as pore size distribution and surface chemistry. For activated carbon materials, for example, capacitance is higher for a sample with wider micropore size distribution than for a sample with higher surface area but too narrow micropore size distribution. In addition, a high amount of surface groups increase the capacitance of a porous electrode, either by improving the wettability of the material or by pseudocapacitance effects. ${ }^{20}$ The poor performance of PEDOTS : DOA 1:10 devices indicates that morphology and porosity of the electrode is not the limiting parameter in this case. Rather, its high hydrophobicity, being a consequence of a higher amount of DOA counterions in the complex, may likely be the cause of a lower degree of swelling and ion uptake in the aqueous electrolyte. ${ }^{4,21}$

To assess the interplay between wettability and porosity of the films with different PEDOTS:DOA ratios, we performed impedance spectroscopy in different background electrolytes, i.e. $0.1 \mathrm{M} \mathrm{KCl}, 0.2 \mathrm{M} \mathrm{KCl}$ and $0.05 \mathrm{M} \mathrm{KCl} / 0.05 \mathrm{M} \mathrm{CaCl}_{2}$ (hereby named $\mathrm{KCl} / \mathrm{CaCl}_{2}$ ), having ionic strengths compatible with biological activity, such as that found in cell culture media.

Compared to the other two compositions, PEDOTS:DOA $1: 1$ revealed a minimized total impedance (Fig. 5a) probably due to a balance between wettability and porosity and a decrease of total impedance with both ionic strength increase and calcium addition. The quantitative analysis of the impedance spectra of the gold electrodes modified with thin films of the complexes was done with the unified equivalent circuit shown in Fig. 5a. The presence of two time-resolved processes could be modelled by two $\mathrm{RC}$ elements in the unified equivalent circuit developed for an electrode covered with a porous coating. ${ }^{22,23}$ Due to the significant film conductivity at applied potentials higher than $0 \mathrm{~V}$, the circuit has been reduced to a simple 4-element equivalent circuit. Since the boundary between the layers is not ideally smooth, a quantitative analysis of the electrode impedance response requires a more complicated, distributed circuit model featuring constant
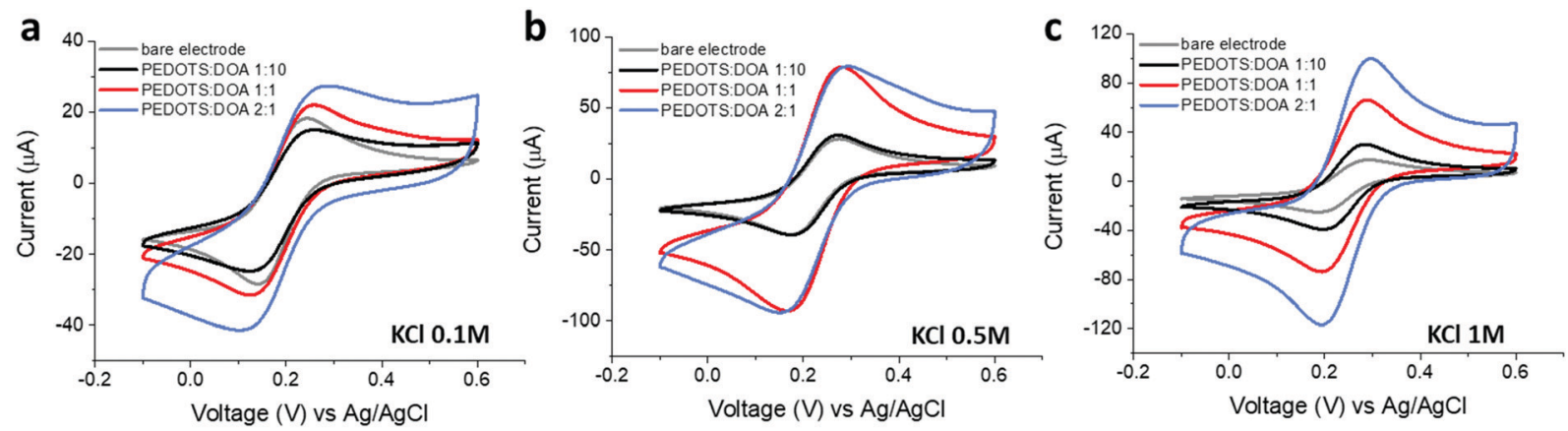

Fig. 3 Cyclic voltammetry of $2 \mathrm{mM} \mathrm{K}_{3}\left[\mathrm{Fe}(\mathrm{CN})_{6}\right]$ in $0.1 \mathrm{M}$ (a), $0.5 \mathrm{M}$ (b) and $1 \mathrm{M}$ (c) $\mathrm{KCl}$ for bare gold IDME and PEDOTS : DOA films at different PEDOTS/ DOA ratios. 

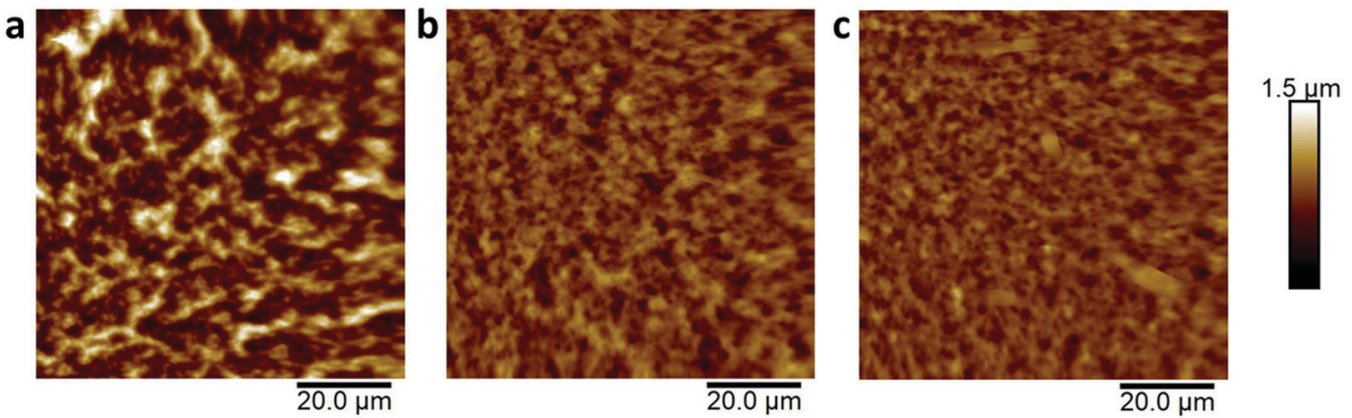

Fig. 4 AFM topography images of dry PEDOTS : DOA 1:10 (a), 1:1 (b) and 2:1 (c) films.

Table 1 Morphology parameters of the PEDOTS:DOA films measured by AFM analysis

\begin{tabular}{|c|c|c|c|}
\hline PEDOTS : DOA & $1: 10$ & $1: 1$ & $2: 1$ \\
\hline Thickness & $325 \mathrm{~nm}$ & $385 \mathrm{~nm}$ & $340 \mathrm{~nm}$ \\
\hline Roughness $R_{\mathrm{RMS}}$ & $219 \mathrm{~nm}$ & $119 \mathrm{~nm}$ & $96 \mathrm{~nm}$ \\
\hline Surface area & $6693 \mu \mathrm{m}^{2}$ & $6572 \mu \mathrm{m}^{2}$ & $6484 \mu \mathrm{m}^{2}$ \\
\hline Surface area difference ${ }^{a}$ & $4.58 \%$ & $2.68 \%$ & $1.32 \%$ \\
\hline
\end{tabular}

${ }^{a}$ The surface area difference represents the ratio between the surface area and the projected area $\left(6400 \mu \mathrm{m}^{2}\right)$, relative to the images in Fig. $4 \mathrm{a}-\mathrm{c}$.

phase elements (CPE) rather than pure capacitors. The capacitance values have been calculated as: ${ }^{24}$

$$
C=\left(P\left(R_{\mathrm{s}}\right)^{(1-\phi)}\right)^{1 / \phi}
$$

where $R_{\mathrm{S}}$ is the solution resistance, $P$ is a fitting parameter of the $\mathrm{CPE}$ and $\phi$ is the fitted exponent factor, which varies from 0 to 1 . When $\phi$ is equal to 0 , the CPE behaves as a pure resistor and when $\phi=1$ the CPE represents a pure capacitor. The equivalent circuit providing the best fit consists of the solution resistance $R_{\mathrm{S}}$ and two parallel branches representing the diffusion through pores (pore resistance $R_{\mathrm{P}}$ and pore capacitance $\left(\mathrm{CPE}_{\mathrm{P}}\right)$ ) and the double layer capacitance $\left(\mathrm{CPE}_{\mathrm{DL}}\right)$. A single set of parameters has been used to simultaneously fit the real and imaginary parts of the impedance spectra over the frequency range from $0.1 \mathrm{~Hz}$ to $50 \mathrm{kHz}$ (Table 2 and Table S1, ESI $\dagger$ ). A value of the fitting quality parameter $\chi^{2}$ of $\leq 0.001$ obtained for all spectra indicates a very good fit. Consistently with total impedance behavior, the PEDOTS : DOA 1:1 showed higher capacitance with respect to the other thin films, and up to 40 times decrease of pore resistance and slight increase of both capacitances with both ionic strength increase and calcium addition showing the ionic transport enhancement. PEDOTS:DOA 1:1 revealed the lowest values of pore resistance and highest capacitance in $0.2 \mathrm{M} \mathrm{KCl}$ and $\mathrm{KCl} / \mathrm{CaCl}_{2}$.

We note that the concept of pore in these soft materials may be problematic. The swelling of the more-or-less hydrophobic complex in electrolytes is a dynamic process and includes formation of "pores" where solvent may insert, thus changing
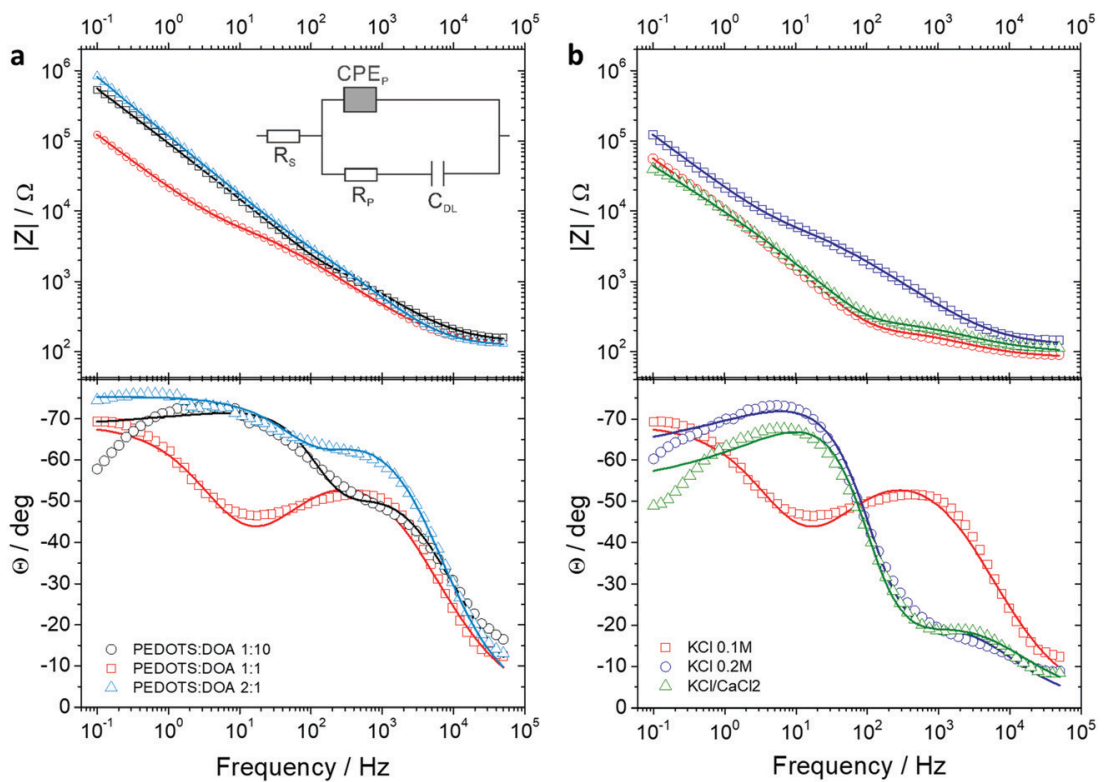

Fig. 5 (a) Impedance spectra acquired on different film-modified gold electrodes in $0.1 \mathrm{M} \mathrm{KCl}$. (b) Impedance spectra of PEDOTS : DOA 1: 1 in different background electrolytes. The solid lines represent the results of the fittings with the equivalent circuit shown in the inset in (a). Bias $0.2 \mathrm{~V}, 10 \mathrm{mV}$ amplitude. 
Table 2 Solution resistance $\left(R_{\mathrm{s}}\right)$, pore resistance $\left(R_{\mathrm{P}}\right)$, pore capacitance $\left(C_{\mathrm{P}}\right)$ and double layer capacitance $\left(C_{\mathrm{DL}}\right)$ of the different films in the different background electrolytes, obtained from the fittings of the impedance spectra of Fig. 5, performed utilizing the unified equivalent circuit in Fig. 5a

\begin{tabular}{|c|c|c|c|c|c|}
\hline PEDOTS : DOA & Electrolyte & $R_{\mathrm{S}}(\mathrm{W})$ & $C_{\mathrm{p}}(\mu \mathrm{F})$ & $R_{\mathrm{p}}(\mathrm{W})$ & $C_{\mathrm{DL}}(\mu \mathrm{F})$ \\
\hline \multirow[t]{3}{*}{$1: 10$} & $0.1 \mathrm{M} \mathrm{KCl}$ & 135 & 0.14 & 3606 & 0.3 \\
\hline & $0.2 \mathrm{M} \mathrm{KCl}$ & 76 & 0.3 & 606 & 1.9 \\
\hline & $0.05 \mathrm{M} \mathrm{KCl}, 0.05 \mathrm{M} \mathrm{CaCl}_{2}$ & 103 & 0.32 & 336 & 2.4 \\
\hline \multirow[t]{3}{*}{$1: 1$} & $0.1 \mathrm{M} \mathrm{KCl}$ & 121 & 0.24 & 7834 & 1.1 \\
\hline & $0.2 \mathrm{M} \mathrm{KCl}$ & 81 & 0.89 & 165 & 5.5 \\
\hline & $0.05 \mathrm{M} \mathrm{KCl}, 0.05 \mathrm{M} \mathrm{CaCl}_{2}$ & 93 & 0.5 & 251 & 4.7 \\
\hline \multirow[t]{3}{*}{$2: 1$} & $0.1 \mathrm{M} \mathrm{KCl}$ & 119 & 0.2 & 8274 & 0.12 \\
\hline & $0.2 \mathrm{M} \mathrm{KCl}$ & 59 & 0.3 & 1821 & 0.62 \\
\hline & $0.05 \mathrm{M} \mathrm{KCl}, 0.05 \mathrm{M} \mathrm{CaCl}_{2}$ & 87 & 0.22 & 518 & 0.23 \\
\hline
\end{tabular}

over time the distribution of pore size and tortuosity. We therefore treat these fitted parameters as very qualitative representation of the steady state geometry of the swollen polymer film.

To monitor the changes in conductance of the PEDOTS : DOA $1: 1$ active layer as a function of the applied voltage in the different electrolytes we then used a four-electrode electrochemical setup comprising two working electrodes as source and drain contacts, a reference electrode and a counter electrode (see inset in Fig. 6a). ${ }^{17,25-28}$ In the three electrolyte solutions, the film showed a transition from a neutral, nonconducting form to an oxidized, highly conducting state, reaching channel resistances 2-3 orders of magnitude lower when changing the electrolyte from $0.1 \mathrm{M} \mathrm{KCl}$ to $\mathrm{KCl} / \mathrm{CaCl}_{2}$ (Fig. 6a). As a result, the corresponding OECTs show greatly improved operation when $\mathrm{KCl} / \mathrm{CaCl}_{2}$ was used as electrolyte (Fig. 6b). Also, while the current increases with the scan rate in $0.1 \mathrm{M} \mathrm{KCl}$, the channel resistance was found to be completely independent on the scan rate in $0.2 \mathrm{M} \mathrm{KCl}$ or in $\mathrm{KCl} / \mathrm{CaCl}_{2}$ electrolyte, suggesting that ions encounter very low resistance to penetrate the bulk film in these conditions.

Improved switching ability of an OECT at increased electrolyte ionic strength has been explained by both a faster ionic motion and the reduction of the Debye length with the concomitant increase in the density of ions that accumulate at the active layer. ${ }^{29}$
Another feature shown in Fig. 6 is a shift of the curves towards more negative voltages when going from $0.1 \mathrm{M} \mathrm{KCl}$ to $0.2 \mathrm{M} \mathrm{KCl}$, and from $0.2 \mathrm{M} \mathrm{KCl}$ to $\mathrm{KCl} / \mathrm{CaCl}_{2}$. Ion sensitive properties of OECTs have been observed in PEDOT:PSS based devices, with the transfer curves shifting to lower gate voltages at increased cations concentrations. The relative change of the gate voltage showed a logarithmic Nernstian dependence on the concentration of the electrolyte, with larger shifts in presence of multivalent cations, in accordance with the largest shift observed for our sample in the presence of bivalent calcium ions. ${ }^{30}$ We cannot exclude also a stabilizing role of the calcium ions when the devices are operated in $\mathrm{KCl} / \mathrm{CaCl}_{2}$ (Fig. S3, ESI $\dagger$ ). Crosslinking of PEDOTS with $\mathrm{Ca}^{2+}$ could indeed cause the polymer precipitation from solutions at $\mathrm{pH}$ above $8,{ }^{13}$ and prevent detachment of thin films from solid substrates. ${ }^{31}$

\section{Experimental}

Poly(4-(2,3-dihydrothieno-[3,4-b]-[1,4]dioxin-2-yl-methoxy)-1butanesulfonic acid, dowex-protonated) PEDOTS was synthesized as previously reported. ${ }^{3}$ Dioctylammonium (DOA) chloride $2.5 \mathrm{mM}$ stock solution was prepared by dissolving dioctylamine (Sigma Aldrich) in $0.1 \mathrm{mM} \mathrm{HCl}$ and adjusting the $\mathrm{pH}$ to 4 (note that $\mathrm{pH}$ and concentration affect both DOA acid/base and
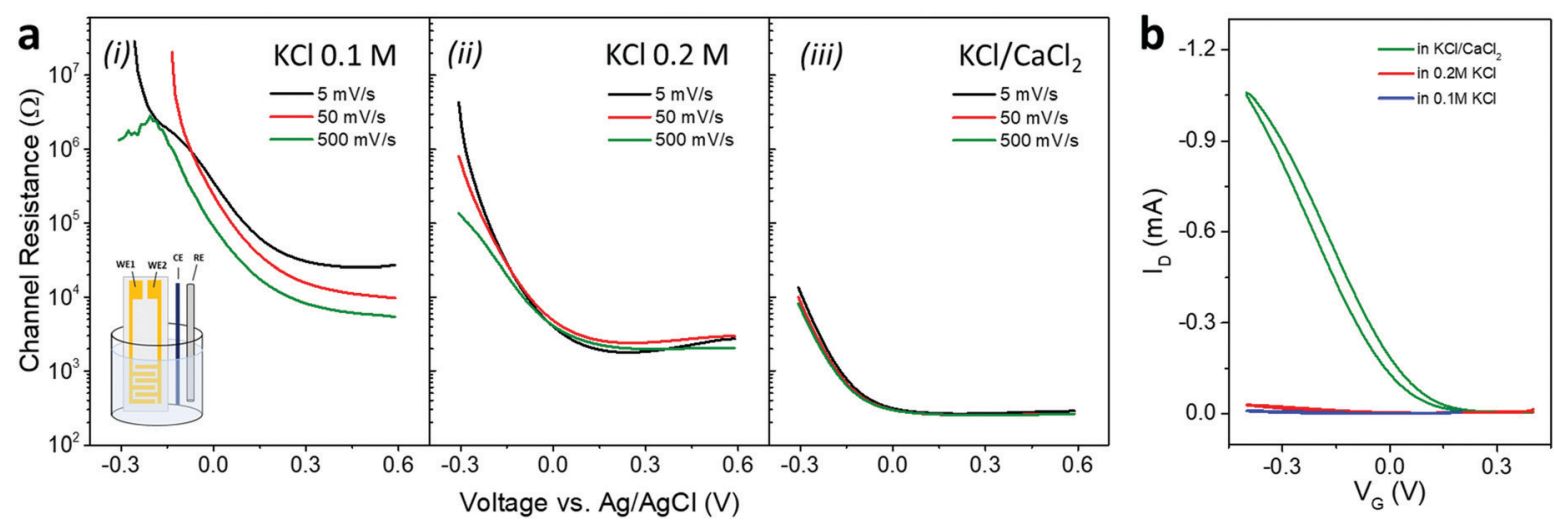

Fig. 6 Performance of PEDOTS: DOA 1:1. (a) Channel resistance as a function of the gate voltage and at different scan rates in a four-electrode setup. The measurements were performed by varying the voltage at the CE (see scheme of the setup in panel (i)) while keeping $50 \mathrm{mV}$ voltage difference between WE1 and WE2. The three panels refer to different electrolytes, as indicated in the legend. (b) Transfer characteristics of OECTs in different electrolytes. 
solubility equilibria and have a big impact on the assembly of the complexes, see also ref. 18). MilliQ water having a resistivity of $18.2 \mathrm{M} \Omega \mathrm{cm}$ was used for preparation of the aqueous solutions. Two-terminal gold interdigitated microelectrodes (IDME) on glass (MicruX Technologies) were used for electrochemical characterizations and device fabrication. All substrates were washed in an ultrasonic bath in water, acetone and isopropanol prior to film deposition.

PEDOTS:DOA complexes having different compositions were obtained by mixing PEDOTS (from $1 \mathrm{mg} \mathrm{mL}^{-1}$ solutions) with DOA at different molar ratios with respect to the EDOTS repeat unit, namely $1: 10,1: 1$ and $2: 1$ (see Fig. 1b). Vortexing for $5 \mathrm{~min}$ resulted in the formation of precipitates of hydrophobic PEDOTS:DOA complexes (Fig. 1c), which could be isolated by successive cycles of centrifugation (10000 rpm, $10 \mathrm{~min}$ ) and washing in $0.1 \mathrm{mM} \mathrm{HCl}$ to remove the excess of DOA or PEDOTS. Finally, the pellets were dried under nitrogen flux for 60 minutes (Fig. 1d). PEDOTS:DOA complexes were dissolved in methanol: chloroform $1: 1$, at a concentration of $1 \mathrm{mg} \mathrm{mL}$ with respect to PEDOTS. For UV-Vis absorbance spectra, all solutions were diluted to $0.05 \mathrm{mg} \mathrm{mL}^{-1}$.

Cyclic voltammetry of the PEDOTS:DOA films deposited on IDME was performed on a Autolab/PGStat10 (Ecochemie, The Netherlands), by using $\mathrm{Ag} / \mathrm{AgCl}$ and a platinum wire as reference and counter electrodes, respectively. $2 \mathrm{mM} \mathrm{K}_{3} \mathrm{Fe}(\mathrm{CN})_{6}$ was used as redox probe, with $\mathrm{KCl}$ at different concentrations as supporting electrolyte.

For the 4-electrode electrochemical measurements, PEDOTS: DOA complexes were drop cast on the IDME on glass. Measurements were performed on an Autolab type III bipotentiostat (Metrohm Autolab, The Netherlands). In this setup, the two gold terminals constituted two working electrodes (WE1 and WE2), while $\mathrm{Ag} / \mathrm{AgCl}(3 \mathrm{M} \mathrm{KCl})$ and a platinum wire were used as reference and counter electrodes, respectively. Measurements were carried out by measuring the current at the working electrodes while changing the voltage at the CE (which corresponds to a gate electrode in the OECT configuration), while keeping the voltage difference between WE1 and WE2 constant. Resistance values were obtained by subtracting the current response at a voltage difference $\Delta V_{\mathrm{WE}}=50 \mathrm{mV}$ with the one at $\Delta V_{\mathrm{WE}}=0 \mathrm{mV}$.

Electrochemical Impedance Spectroscopy (EIS) of the PEDOTS:DOA films deposited on IDME was performed on an Autolab type III bipotentiostat (Metrohm Autolab, The Netherlands).

Electrochemical transistors (OECTs) were fabricated by drop casting few microliters of the PEDOTS:DOA complexes in chloroform/methanol on the IDME and using $\mathrm{Ag} / \mathrm{AgCl}$ as gate electrode. Potassium chloride $(\mathrm{KCl})$ and calcium chloride $\left(\mathrm{CaCl}_{2}\right)$ at different concentrations and ratios were used as electrolytes. A scheme of the devices is shown in Fig. 2b. The electrical characterization of the devices was performed on a Keithley 4200 parameter analyzer, in air and at room temperature. The measurements were performed at a scan rate of $20 \mathrm{mV} \mathrm{s}^{-1}$. All the transistors had the same channel length $(L=20 \mu \mathrm{m})$ and width $(W=39000 \mu \mathrm{m})$.

Atomic force microscopy (AFM) of the dried films was performed in tapping mode with a Dimension 3100/Nanoscope
IIIa system, by using commercial silicon cantilevers with a nominal spring constant of $40 \mathrm{~N} \mathrm{~m}^{-1}$. Films thicknesses measured by AFM on representative devices were $325 \mathrm{~nm}$ for the $1: 10$, $385 \mathrm{~nm}$ for the $1: 1$ and $340 \mathrm{~nm}$ for the $2: 1$ PEDOTS: DOA films.

\section{Conclusions}

We have shown that ionic transport in PEDOTS based complexes is governed by a delicate balance among degree of doping, wettability and porosity, and that these parameters can be controlled by engineering the supramolecular assembly through a precise tuning of the polyelectrolyte/hydrophobic counterion ratio. We have also shown that is possible to improve OECT operation by slightly increasing the ionic strength of the electrolyte or by adding bivalent calcium ions, while maintaining it in a range compatible with biological operation. This material is therefore suitable to operate at cell interfaces.

\section{Conflicts of interest}

There are no conflicts to declare.

\section{Acknowledgements}

This work was supported by the Knut and Alice Wallenberg foundation, through a Wallenberg Scholar grant to O. I., and Advanced Functional Materials (AFM) at Linköping University a Swedish Government Strategic Research Area.

\section{References}

1 D. T. Simon, E. O. Gabrielsson, K. Tybrandt and M. Berggren, Organic Bioelectronics: Bridging the Signaling Gap between Biology and Technology, Chem. Rev., 2016, 116, 13009-13041.

2 A. O. Patil, Y. Ikenoue, N. Basescu, N. Colaneri, J. Chen, F. Wudl and A. J. Heeger, Self-Doped Conducting Polymers, Synth. Met., 1987, 20, 151-159.

3 R. H. Karlsson, A. Herland, M. Hamedi, J. A. Wigenius, A. Åslund, X. Liu, M. Fahlman, O. Inganäs and P. Konradsson, Iron-Catalyzed Polymerization of AlkoxysulfonateFunctionalized 3,4-Ethylenedioxythiophene Gives WaterSoluble Poly(3,4-Ethylenedioxythiophene) of High Conductivity, Chem. Mater., 2009, 21, 1815-1821.

4 A. Laiho, L. Herlogsson, R. Forchheimer, X. Crispin and M. Berggren, Controlling the Dimensionality of Charge Transport in Organic Thin-Film Transistors, Proc. Natl. Acad. Sci. U. S. A., 2011, 108, 15069-15073.

5 J. Rivnay, S. Inal, A. Salleo, R. M. Owens, M. Berggren and G. G. Malliaras, Organic Electrochemical Transistors, Nat. Rev. Mater., 2018, 3, 17086.

6 E. Zeglio and O. Inganäs, Active Materials for Organic Electrochemical Transistors, Adv. Mater., 2018, e1800941.

7 M. Braendlein, T. Lonjaret, P. Leleux, J. M. Badier and G. G. Malliaras, Voltage Amplifier Based on Organic Electrochemical Transistor, Adv. Sci., 2017, 4, 1600247. 
8 M. Ghittorelli, L. Lingstedt, P. Romele, N. I. Craciun, Z. M. Kovacs-Vajna, P. W. M. Blom and F. Torricelli, High-Sensitivity Ion Detection at Low Voltages with Current-Driven Organic Electrochemical Transistors, Nat. Commun., 2018, 9, 1441.

9 A. Elfwing, F. G. Bäcklund, C. Musumeci, O. Inganäs and N. Solin, Protein Nanowires with Conductive Properties, J. Mater. Chem. C, 2015, 3, 6499-6504.

10 F. G. Backlund, A. Elfwing, C. Musumeci, F. Ajjan, V. Babenko, W. Dzwolak, N. Solin and O. Inganas, Conducting Microhelices from Self-Assembly of Protein Fibrils, Soft Matter, 2017, 13, 4412-4417.

11 C. Müller, M. Hamedi, R. Karlsson, R. Jansson, R. Marcilla, M. Hedhammar and O. Inganäs, Woven Electrochemical Transistors on Silk Fibers, Adv. Mater., 2011, 23, 898-901.

12 C. Muller, R. Jansson, A. Elfwing, G. Askarieh, R. Karlsson, M. Hamedi, A. Rising, J. Johansson, O. Inganas and M. Hedhammar, Functionalisation of Recombinant Spider Silk with Conjugated Polyelectrolytes, J. Mater. Chem., 2011, 21, 2909-2915.

13 M. Hamedi, A. Elfwing, R. Gabrielsson and O. Inganäs, Electronic Polymers and DNA Self-Assembled in Nanowire Transistors, Small, 2013, 9, 363-368.

14 C. A. Cutler, M. Bouguettaya and J. R. Reynolds, Pedot Polyelectrolyte Based Electrochromic Films Via Electrostatic Adsorption, Adv. Mater., 2002, 14, 684.

15 C. A. Cutler, M. Bouguettaya, T.-S. Kang and J. R. Reynolds, Alkoxysulfonate-Functionalized Pedot Polyelectrolyte Multilayer Films: Electrochromic and Hole Transport Materials, Macromol. Chem., 2005, 38, 3068.

16 M. Hamedi, J. Wigenius, F.-I. Tai, P. Bjork and D. Aili, Polypeptide-Guided Assembly of Conducting Polymer Nanocomposites, Nanoscale, 2010, 2, 2058-2061.

17 E. Zeglio, J. Eriksson, R. Gabrielsson, N. Solin and O. Inganäs, Highly Stable Conjugated Polyelectrolytes for Water-Based Hybrid Mode Electrochemical Transistors, Adv. Mater., 2017, 29, 1605787.

18 P. K. Johansson, D. Jullesson, A. Elfwing, S. I. Liin, C. Musumeci, E. Zeglio, F. Elinder, N. Solin and O. Inganäs, Electronic Polymers in Lipid Membranes, Sci. Rep., 2015, 5, 11242.

19 C. Punckt, M. A. Pope and I. A. Aksay, On the Electrochemical Response of Porous Functionalized Graphene Electrodes, J. Phys. Chem. C, 2013, 117, 16076-16086.

20 D. Lozano-Castelló, D. Cazorla-Amorós, A. Linares-Solano, S. Shiraishi, H. Kurihara and A. Oya, Influence of Pore Structure and Surface Chemistry on Electric Double Layer Capacitance in Non-Aqueous Electrolyte, Carbon, 2003, 41, 1765-1775.
21 A. Giovannitti, D.-T. Sbircea, S. Inal, C. B. Nielsen, E. Bandiello, D. A. Hanifi, M. Sessolo, G. G. Malliaras, I. McCulloch and J. Rivnay, Controlling the Mode of Operation of Organic Transistors through Side-Chain Engineering, Proc. Natl. Acad. Sci. U. S. A., 2016, 113, 12017-12022.

22 M. O'Donoghue, R. Garrett, V. Datta, P. Roberts and T. Aben, Electrochemical Impedance Spectroscopy: Testing Coatings for Rapid Immersion Service, Mater. Perform., 2003, 42, 36-41.

23 D. Loveday, P. Peterson and B. Rodgers, Evaluation of Organic Coatings with Electrochemical Impedance Spectroscopy - Part 3: Protocols for Testing Coatings with Eis, JCT CoatingsTech, 2005, 2, 22-27.

24 V. D. Jović and B. M. Jović, Eis and Differential Capacitance Measurements onto Single Crystal Faces in Different Solutions: Part I: $\mathrm{Ag}(111)$ in $0.01 \mathrm{M} \mathrm{Nacl,} \mathrm{J.} \mathrm{Electroanal.} \mathrm{Chem.,}$ 2003, 541, 1-11.

25 E. Peintler-Kriván, P. S. Tóth and C. Visy, Combination of in Situ Uv-Vis-Nir Spectro-Electrochemical and A.C. Impedance Measurements: A New, Effective Technique for Studying the Redox Transformation of Conducting Electroactive Materials, Electrochem. Commun., 2009, 11, 1947-1950.

26 E. Zeglio, M. Vagin, C. Musumeci, F. N. Ajjan, R. Gabrielsson, X. T. Trinh, N. T. Son, A. Maziz, N. Solin and O. Inganäs, Conjugated Polyelectrolyte Blends for Electrochromic and Electrochemical Transistor Devices, Chem. Mater., 2015, 27, 6385-6393.

27 J. W. Thackeray, H. S. White and M. S. Wrighton, Poly(3-Methylthiophene)-Coated Electrodes: Optical and Electrical Properties as a Function of Redox Potential and Amplification of Electrical and Chemical Signals Using Poly(3-Methylthiophene)Based Microelectrochemical Transistors, J. Phys. Chem., 1985, 89, 5133-5140.

28 G. Schiavon, S. Sitran and G. Zotti, A Simple Two-Band Electrode for in Situ Conductivity Measurements of Polyconjugated Conducting Polymers, Synth. Met., 1989, 32, 209-217.

29 J. T. Mabeck, J. A. DeFranco, D. A. Bernards, G. G. Malliaras, S. Hocdé and C. J. Chase, Microfluidic Gating of an Organic Electrochemical Transistor, Appl. Phys. Lett., 2005, 87, 013503.

30 P. Lin, F. Yan and H. L. W. Chan, Ion-Sensitive Properties of Organic Electrochemical Transistors, ACS Appl. Mater. Interfaces, 2010, 2, 1637-1641.

31 K. M. Persson, R. Gabrielsson, A. Sawatdee, D. Nilsson, P. Konradsson and M. Berggren, Electronic Control over Detachment of a Self-Doped Water-Soluble Conjugated Polyelectrolyte, Langmuir, 2014, 30, 6257-6266. 